\title{
Vaccin contre le virus du papillome humain : en attente d'un miracle
}

$\mathrm{L}$ e JAMC publie ce numéro spécial sur le vaccin contre le virus du papillome humain (VPH) en partie pour reconnaître les chercheurs qui ont mis au point le vaccin et l'industrie pharmaceutique qui s'est lancée dans la tâche longue et difficile de la commercialisation de ces vaccins. L'industrie n'a jamais considéré les vaccins comme lucratifs. Dans l'optique de la santé publique, toutefois, ils ont évité ou éradiqué de nombreuses maladies. Le lancement du vaccin contre la polio au Canada en 1955 a mis fin à l'horreur des éclosions annuelles. Conjugués aux programmes de dépistage du cancer du col, les vaccins contre le VPH peuvent maintenant transformer en mauvais souvenir les décès causés par ce cancer. Dans ce numéro, nous résumons les données existantes, déterminons le nombre de personnes à vacciner et discutons de préoccupations reliées aux politiques (Encadré I).

Il reste encore beaucoup à faire. Malgré un excellent départ, beaucoup de questions sont encore sans réponse, notamment l'efficacité à long terme, la dose optimale, l'efficacité globale contre le VPH dans le monde réel et les modes de distribution optimaux dans les populations à risque et démunies ${ }^{1}$.

Même s'il existe un optimisme prudent au sujet des avantages à long terme que pourraient offrir les deux nouveaux vaccins contre le $\mathrm{VPH}$, on craint de plus en plus que les efforts de mise au point des vaccins ne soient relégués aux oubliettes en faveur de produits pharmaceutiques plus rentables. Le coût élevé de la mise au point des vaccins et les marchés limités, conjugués à l'inquiétude que soulèvent les vaccins chez certains, ont rendu peu attrayant le développement de vaccins, particulièrement face à des médicaments liés aux «habitudes de vie» comme le sildénafil — qui produit inévitablement des recettes à cause de son utilisation régulière généralisée. Étant donné l'importance de l'investissement, une poignée seulement de grandes entreprises demeurent vouées à la recherche-développement sur les vaccins. Or, comme en témoigne la saga de la polio, les vaccins ont démontré qu'ils peuvent être d'une efficacité remarquable pour prévenir les maladies et réduire la souffrance humaine.

Les vaccins contre le VPH sont porteurs d'une promesse semblable. Ils auront toutefois besoin d'aide. Le cancer du col a une prédilection pour les femmes pauvres et démunies des pays industrialisés et des pays en développement. En Occident, les programmes de vaccination et de dépistage du cancer du col chez les personnes à risque élevé comme les jeunes autochtones, les jeunes itinérantes et les personnes des régions démunies présentent des obstacles importants à l'éradication du cancer du col. Dans le monde, plus de $80 \%$ des décès attribuables au cancer du col se produisent dans les pays en développement, où l'on confirme 470000 cas par année (environ la moitié du nombre des nouveaux cas de cancer du sein) ${ }^{2}$.

Il ne faut pas oublier les pays en développement. Il ne faut pas que les retards et les obstacles qui ont surgi dans la lutte visant à éradiquer la polio en Afrique et en Asie du Sud-Est se répètent dans la lutte contre le cancer du col avec le vaccin contre le VPH et les programmes de dépistage de ce cancer. Cette lutte nécessitera plus que de l'argent et des vaccins : il faudra des stratégies de mise en œuvre particulières à chaque pays, du leadership, de l'infrastructure et une sensibilisation culturelle. Les grandes sociétés pharmaceutiques ont beaucoup à apporter parce qu'elles ont abondamment de leadership, d'expertise en gestion et en logistique et de main-d'œuvre administrative. Le JAMC

\section{Encadré 1 : Dans ce numéro du JAMC}

- Brisson et ses collègues estiment que le nombre de personnes à vacciner contre l'infection au virus du papillome humain (VPH) serait de 8 pour prévenir un épisode de condylomes et de 324 pour prévenir un cas de cancer du col (page 464).

- Un examen systématique réalisé par Rambout et ses collègues révèle que la vaccination prophylactique contre le VPH est très efficace pour prévenir l'infection au VPH propre au type de vaccin ainsi que les maladies du col de l'utérus. Les auteurs signalent toutefois un certain nombre de limites au niveau de la conception de l'étude et des lacunes dans les connaissances (page 469).

- Ogilvie et ses collègues démontrent la possibilité d'une auto-collecte des spécimens pour le test de dépistage du VPH chez les femmes qui pourraient ne pas utiliser les programmes de dépistage cytologique et qui courent donc un risque accru de développer un cancer du col (page 480).

- Lippman et ses collègues s'élèvent contre la mise en œuvre généralisée des programmes de vaccination. Ils déclarent qu'il n'y a pas d'épidémie et qu'il n'est donc pas urgent de mettre en œuvre un programme de vaccination massive (page 484).

- Dawar et ses collègues examinent l'épidémiologie et les répercussions en termes de maladie de l'infection au VPH et décrivent les études cliniques sur deux vaccins contre le VPH (page 456).

- Un feuillet d'information pour les patients au sujet du VPH est fourni (page 462).

implore l'industrie des vaccins de faire généreusement don de son temps et de son expertise pour créer un miracle. Nous encourageons les entreprises à collaborer avec des intervenants clés, y compris les autorités locales, les organismes non gouvernementaux et les bailleurs de fonds, afin que l'on puisse distribuer des vaccins contre le papillomavirus et lancer des programmes de dépistage du cancer du col dans les pays en développement. Un miracle est possible : les outils (vaccins et dépistage) sont là. Il est possible de vaincre le cancer du col.

\section{Noni MacDonald MD MSc}

Rédactrice de section, Santé publique, JAMC

Paul C. Hébert MD MHSc

Rédacteur en chef, JAMC

Pour l'équipe de rédaction de l'éditorial (Matthew Stanbrook, Barbara Sibbald, Ken Flegel et Amir Attaran)

\section{RÉFÉRENCES}

I. Agence de la santé publique du Canada. Atelier sur les priorités canadiennes en matière de recherche sur les vaccins contre le virus du papillome humain : Rapport final. RMTC 2006;32(suppl. I). Disponible à l'adresse : www.phac-aspc.gc.ca/publicat /ccdr-rmtc/o6vol32/32si/index.html (consulté le 26 juillet 2007).

2. Stewart BW, Kleihues P. Le cancer dans le monde. Genève : OMS; 2003. Disponible à l'adresse : www.iarc.fr/IARCPress/pdfs/wcrfl (consulté le 26 juillet 2007). 